\title{
Star Formation in Infrared Bright and Infrared Faint Starburst Interacting Galaxies.
}

\author{
Susan A. Lamb, ${ }^{\dagger \dagger}$ Howard A. Bushouse, ${ }^{\S}$ and John W. Towns*
}

\begin{abstract}
.
Short wavelength IUE spectra of Arp 248b and UGC $8315 \mathrm{~N}$ are combined with optical spectra and interpreted using a combination of spectrum synthesis and spectral diagnostics to place constraints on the massive star populations of the central regions of these galaxies and to deduce information about the star formation histories in the last $10^{8}$ years. We find that both galaxies have substantial fractions of their optical light coming from massive stars and that Arp 248b may be dominated in the UV by WR stars. The UV spectra are dominated by radiation from evolved massive stars and we place an age on the burst in Arp 248b of a few tens of millions of years.
\end{abstract}

\section{Introduction.}

The stellar content and star formation histories of interacting and merging galaxies are of particular interest because they are of relevance to many aspects of the evolution of these systems. For example, the number of and distribution with mass of massive stars produced will effect the eventual supernova rate and return of energy and material to the interstellar media. We need to know the stellar population produced if we wish to have an understanding of the processes that occur when two galaxies collide, for example, the amount of mass going into massive versus low mass stars will influence the total infrared flux of the galaxies. Ultraviolet radiation from galaxies comes predominantly from the massive star population $(\mathrm{M}>10 \mathrm{MO})$ and thus provides a useful probe of this end of the mass spectrum, although dust obscuration in the UV is very high and has to be taken into account when interpreting observations. Because of the complications introduced by the pesence of dust, the general method of interpreting UV spectra relies on the use of spectral diagnostics, such as the shape and depth of the Si IV and C IV lines, rather than detailed spectrum synthesis and is most useful when the UV data is complimented by spectrum synthesis of optical spectra of the same region of the object. Here we report on a preliminary investigation, using this method, of the massive star content of two galaxies for which we already had optical data. We obtained UV (IUE) spectra of UGC $8315 \mathrm{~N}$ and Arp 248b, which are both high star formation rate galaxies as observed from their optical spectra, and members of strongly interacting binary systems. They were chosen from the Bushouse (1986) sample of closely interacting galaxies and as such were selected to show optical morphological evidence of prior interaction in the combined system, i.e., optical photographs show evidence of bridges and tails.

\section{Interacting Galaxies: Data and Subsamples.}

The study of the UV properties of interacting galaxies is part of a larger project to investigate the star 
formation histories and stellar content of these systems using observations in various wavelength regions as well as theoretical modelling (see Gerber, Balsara, and Lamb, this volume). The Bushouse sample, from which our two galaxies were chosen, has been the focus of these investigations. Nuclear region optical spectra (Bushouse, 1986b) and global $\mathrm{H} \alpha$ fluxes (Bushouse, 1987) together with IRAS observations (Bushouse, Lamb, and Werner, 1988) have shown a large span of stellar population mixes among the sample members, ranging from strong stellar burst like to elliptical galaxy like populations. This indicates a wide range in star formation activity over the last billion years.

One important result found by Bushouse, Lamb, and Werner (1988) for the strongly interacting systems is that they can be subdivided into at least five groups based upon their optical and far infrared (IRAS) properties. Some of these properties are listed in Table 1 for the five groups and for the Ultraluminous IRAS galaxies studies by Sanders et al. (1988), as a comparison. We note that the value of $L_{\mathbb{I R}} / L_{B}$ increases from a value of 2.5 for the low star formation rate galaxies to one of 162 for the ultraluninous systems. Also at the low end is a group of high star formation, low infrared flux systems. These consist of low mass, dwarf galaxies and presumably contain little dust, however optical observations indicate high rates of star formation. In this paper we concentrate on sample members of two of these groups; UGC $8315 \mathrm{~N}$ is a member of the 'High SFR-Low IR' group, and Arp 248b is a member of the 'High SFR' group.

Table 1

MEAN PROPERTIES OF SUBSAMPLES

\begin{tabular}{llclc}
\hline \hline SAMPLE & $\mathrm{L}_{\mathrm{IR}}\left(10^{9} \mathrm{LO}\right)$ & $\mathrm{LB}_{\mathrm{B}}\left(10^{9} \mathrm{LO}\right)$ & $\mathrm{L}_{\mathrm{IR}} / \mathrm{L}_{\mathrm{B}}$ & $\log \left(\mathrm{f}_{60} / \mathrm{f}_{100}\right)$ \\
\hline \hline Low SFR & 12. & 3.8 & 2.5 & -0.55 \\
High SFR- Low IR* & 1. & 0.4 & 2.5 & -0.32 \\
Disk SF & 36. & 3.9 & 9.3 & -0.35 \\
High SFR* & 34. & 1.4 & 14.2 & -0.21 \\
High LIR/LB & 118. & 2.4 & 54.0 & -0.15 \\
Ultraluminous & 766. & 8.5 & 162.0 & +0.02 \\
IRAS galaxies $\dagger$ & & & & \\
\hline \hline
\end{tabular}

*We here study galaxies from these two subsamples.

†From Sanders et al. (1988) 


\section{IUE and Optical Spectra.}

The two galaxies studied here show strong evidence from their optical spectra of high levels of current star formation and hence are classified as starburst galaxies, but they differ in that Arp 248b has a large far infrared luminosity of $2.225 \times 10^{10} \mathrm{LO}$ and a $\mathrm{L}_{\mathrm{IR}} / \mathrm{L}_{\mathrm{H} \alpha}$ of 333 , whereas UGC $8315 \mathrm{~N}$ is barely detected by IRAS. The two galaxies are thus representative of different classes of starburst galaxies (as discussed above). UGC $8315 \mathrm{~N}$ is in a system of two dwarf galaxies in contact and with tails, whereas Arp $248 \mathrm{~b}$ is one of a pair of spirals connected by a bridge and also having tails. Some properties of the two galaxies are given in Table 2.

Table 2

GALAXY PROPERTIES

\begin{tabular}{lll}
\hline \hline Galaxy & UCG $8315 \mathrm{~N}$ & $\operatorname{Arp~} 248 \mathrm{~b}$ \\
\hline \hline Distance & $16 \mathrm{Mpc}$ & $68 \mathrm{Mpc}$ \\
Ang. size & $15^{\prime \prime} \times 10^{\prime \prime}$ & $40^{\prime \prime} \times 30^{\prime \prime}$ \\
$\mathrm{L}_{\mathrm{B}}$ & $2.7 \times 10^{7} \mathrm{LO}$ & $1.61 \times 10^{9} \mathrm{LO}$ \\
$\mathrm{L}_{\mathrm{IR}}$ & $<8 \times 10^{7} \mathrm{LO}$ & $22.25 \times 10^{9} \mathrm{LO}$ \\
$\mathrm{L}_{\mathrm{B} / \mathrm{L} / \mathrm{R}}$ & $<1.8$ & 13.8 \\
$\mathrm{H}_{\alpha}(\mathrm{EW})$ & $190 \mathrm{~A}$ & $62.8 \mathrm{~A}$ \\
$\mathrm{E}(\mathrm{B}-\mathrm{V})$ & 0.10 & 0.47 \\
$\mathrm{AV}$ & 0.32 & 1.4 \\
$\mathrm{O} / \mathrm{H}$ & $2 . \times 10^{-4}$ & $6.5 \times 10^{-4}(\sim$ solar $)$ \\
\hline \hline
\end{tabular}

The IUE ultraviolet observations were obtained on DATE. They are short wavelength (1150 - 1950 A) and of low dispersion ( $~ 6 \mathrm{~A} / \mathrm{mm}$.). The large aperture (10" $\left.\times 20^{\prime \prime}\right)$ was used for both observations and covers the whole of UGC $8315 \mathrm{~N}$ but only the nucleus of Arp 248b. The exposure times for the two galaxies respectively were 10.4 and 14.28 hours. The exposure on UGC $8315 \mathrm{~N}$ was shortened by observational constraints and was marred by a high dispersion residual from a previous overexposure of the camera. The resulting spectra is thus of low signal to noise. However, enough information is available to provide a rough comparison with the spectrum of Arp $248 \mathrm{~b}$ which has a signal to noise of $175 / 105$. The ultraviolet spectra of the two galaxies is shown in Figure 1 . and is superimposed on a model fit which is discussed below.

The optical observations of UGC $8315 \mathrm{~N}$ were obtained in April, 1984 at Steward Observatory on the $90 "$ telescope with the reticon spectrograph: the aperture was 5" in diameter. Those of Arp 248b were obtained in the same month at Kitt Peak using the IRS (intensified reticon spectrograph): the aperture was 22 " in diameter. Both observations covered the galactic nuclei on 

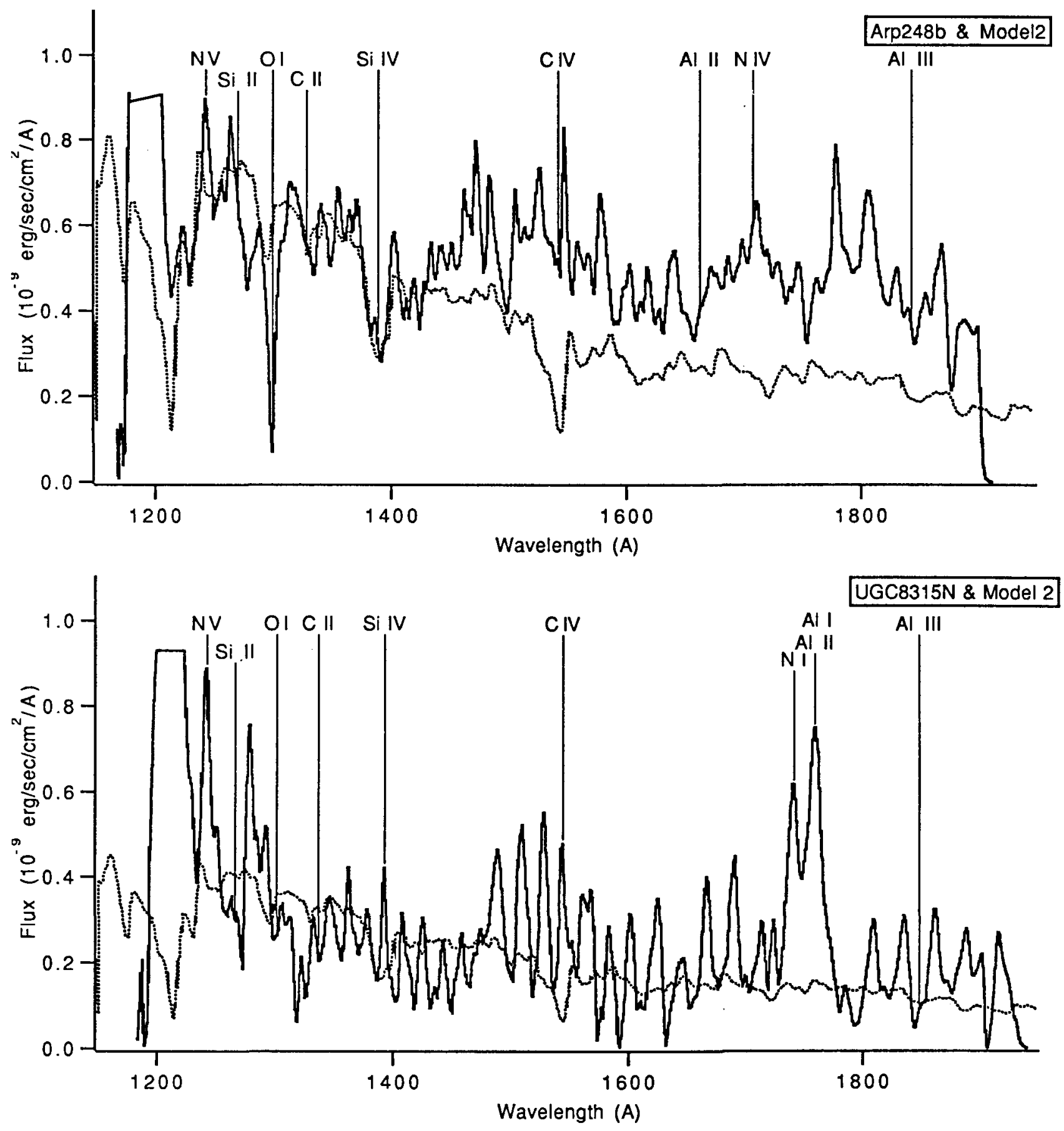

Figure 1. The IUE spectra of Arp 248b and UGC 8315N (dark lines) and a model fit (dotted lines). Arp 248b appears to have a large component of WR stars giving rise to the emission humps between 1450 and $1900 \mathrm{~A}$. 


\section{Population Synthesis and Interpretation.}

The spectrum of Arp 248b is of sufficiently good signal to noise that absorption lines of O I ( $\lambda 1302$ A), Si IV $(\lambda 1394,1403 \mathrm{~A}), \mathrm{C}$ IV $(\lambda 1548,1551 \mathrm{~A})$, and Al III $(\lambda 1855 \mathrm{~A})$ are well observed. However in UGC $8315 \mathrm{~N}$ only emission lines of $\mathrm{N} \mathrm{V}(\lambda 1239,1243 \mathrm{~A}), \mathrm{N}$ I $(\lambda 1743,1745 \mathrm{~A})$, and $\mathrm{Al}$ I/II $(\lambda 1766-1776 \mathrm{~A})$ are easily identified. Comparison between the UV spectrum of Arp $248 \mathrm{~b}$ and the best model fit (discussed below) indicates the presence of emission humps between 1450 and 1900 A similar to those found in WN8 UV spectra (see Nussbaumer et al. 1982).

Concentrating on the spectrum of Arp 248b, we note that the lack of significant C IV absorption implies the absence of any large component of massive main sequence stars, and C IV emission (if present) implies very hot massive stars. The Si IV absorption is moderately strong and there is a hint of a P Cygni profile. This implies the presence of evolved massive stars, ie. supergiants. The very strong $\mathrm{O} I$ absorption line can indicate the presence of main sequence $B$ stars. This line is found to be strongest for the B5 V class. Lastly, the emission humps strongly suggest the presence of a substantial number of WR stars.

Spectrum synthesis of the optical spectra of UGC $8315 \mathrm{~N}$ and Arp $248 \mathrm{~b}$ indicate that OB stars contribute $\sim 50 \%$ and $~ 30 \%$ of the optical radiation, respectively. However, the UV spectra are not fit by any normal models based on the Wu et al.(1983) IUE spectral atlas of stars. (This atlas does not include WR stellar spectra). We compared the observed spectra with models covering a range of parameters, including age of population, high mass cutoff of the initial mass function, and spread in creation times of the individual stars in the population (for a discussion of the set of models see Lamb, Hunter, and Gallagher, 1990). The best fit for Arp 248b was model 2 (displayed in figure 1) which is for a single stellar burst of age 3 million years. The fit is not sensitive to the upper mass cutoff of the initial mass function. Model 2 is also used to compare with the spectrum of UGC $8315 \mathrm{~N}$, although most of the models can be fit to this spectrum as it has poor signal to noise. This comparison helps provide the evidence for a large population of WR stars in Arp 248b but not in UGC $8315 \mathrm{~N}$. The flat UV spectra found in both cases is similar to those often observed for 'starburst' nuclei and is interpreted as due to massive hot stars. The UV spectrum of Arp 248b is not unique and has similarities to that of DDO 50, a dwarf galaxy dominated by WR stars (see Lamb, Hunter and Gallagher, 1990). WR stars have also been detected in other powerful far-infrared, starburst galaxies (see Armus, Heckman, and Miley, 1988).

A direct interpretation of the optical and UV data for Arp 248b would give a picture of the galaxy as having experienced a 'starburst' at least a few million years ago. Evidence of continuing star formation is not present in the UV spectrum. The maximum age of the burst if just one, of short duration, occurred, is a few tens of millions of years. However, there are complications involving the dust. The extinction in the UV is sufficiently large that we may be viewing only those massive stars that have 'broken out' of their natal gas and dust. This would preclude observation in the UV of the youngest massive stars and could hide current and recent star formation. It would complicate comparisons between observations of galaxies in which dust is clumped differently, or in which the amount of dust is very different. We do not anticipate that dust would obscure most WR stars, however, and we thus conclude that the difference between our two program galaxies in this respect is real. 


\section{Conclusions.}

We conclude that short wavelength IUE spectra of colliding galaxies supports the idea that large numbers of massive stars $\left(\mathrm{M}>20 \mathrm{M}_{\mathrm{O}}\right)$ are produced in bursts. The massive stellar populations may be somewhat different in the two galaxies studied here. That in UGC $8315 \mathrm{~N}$ may be hotter than in Arp 248b. The optical spectra support this. (However metallicity differences may confuse the issue). We also find evidence that Arp $248 \mathrm{~b}$ contains many WR stars, unlike UGC $8315 \mathrm{~N}$. We are observing evolved massive stars in the UV. This may simply reflect ageing single bursts of star formation, or it may be due to preferential obscuration of young stars by dust.

\section{References.}

Armus, L., Heckman, T. M., and Miley, G. K. (1988) Ap.J., 326, L45.

Bushouse, H. A. (1986) Ph.D. thesis, University of Illinois.

Bushouse, H. A. (1986) A.J., 91, 255; 1987.

Bushouse, H. A. (1987) Ap.J., 320, 49.

Bushouse, H. A., Lamb, S. A., and Werner, M. W. (1988) Ap.J., 335, 74.

Lamb, S. A., Hunter, D. A., and Gallagher, J. S. (1990) submitted to Ap. J.

Nussbaumer, H., Schmutz, W., Smith, L. J., and Willis, A. J. (1982) Astron. Astrophs. Suppl., 47, 257.

Sanders, D. B., Soifer, B. T., Elias, J. H., Madore, B. F., Matthews, K., Neugebauer, G., and Scoville, N. Z. (1988) Ap.J., 325, 74.

Wu, C. -C. et al. (1983) IUE Ultraviolet Spectral Atlas, IUE NASA Newsletter No. 22.

* Department of Physics, University of Illinois , Urbana, Illinois 61801.

† Department of Astronomy, University of Illinois , Urbana, Illinois 61801.

$\S$ Department of Physics, Northwestern University, Evanston, Illinois 60201 